\title{
Naciones mediáticas. Los marcos informativos de la consulta sobre la independencia de Cataluña (9-N)
}

\author{
Media nations. News framing of the Catalonian independence \\ referendum $(9-N)$
}

\author{
Carlos A. Ballesteros Herencia. Universidad de Valladolid
}

Recibido: 4-XI-2014 - Aceptado: 12-II-2015

Resumen:

La propuesta de celebrar un referéndum sobre la independencia de Cataluña ha recibido una intensa representación informativa en los medios españoles, como correlato de la continua actividad política que ha supuesto. Para contrastar la hipótesis de que los medios catalanes y madrileños realizaran un tratamiento diferente de esta cuestión, se planteó describir los componentes esenciales de los marcos mediáticos utilizados por los principales periódicos sobre este hecho informativo: fuentes, actores protagonistas, acciones descritas y evaluación. Para ello, esta investigación se propuso aplicar la metodología propia de la teoría del framing o enmarcado informativo, realizando un estudio cuantitativo apoyado en la aplicación de la estadística, sobre una muestra de los diarios El País, El Mundo, ABC, La Vanguardia, El Periódico y El Punt Avui. Junto al cálculo de estadísticos descriptivos, se halló también la existencia o no de asociación significativa entre variables. Este tema apareció en portada todos los días en la mitad o más de los medios analizados, con una importancia formal entre alta y máxima. Las principales fuentes y protagonistas fueron las instituciones y los políticos, realizando acciones de tipo político. La prensa madrileña y la catalana evaluaron de modo contrario las noticias protagonizadas por las instituciones catalanas y españolas.

Palabras clave:

Marcos informativos, información política, consulta, referéndum, Cataluña.

Abstract:

The proposal to hold a referendum on the independence of Catalonia has been the subject of intense news coverage in the Spanish media, similar to the constant political activity. This research aimed to apply the methodology of news framing, making a quantitative study supported on the application of statistics. It was posed to describe the main components of media frames used to report on this informative fact: news sources, protagonists, described actions and evaluation of the news event. Also, we wanted to know, as hipothesis, if the press from Madrid and Catalonia offered a different news framing about this issue. Beside the calculation of descriptive statistics for these variables, it was also calculated the existence of a statistically significant association between variables. A sample of the main spanish journals was selected: El País, El Mundo, ABC, La Vanguardia, El Periódico y El Punt Avui. This topic appeared on the front page of the newspapers analyzed every day, with a formal importance from high to maximum. The main sources and protagonists were the institutions and the politicians, performing political actions. The press from Madrid and Catalonia evaluated in an opposite way the news where the Catalonian or Spanish institutions were the protagonists.

Keywords:

News frames, political news, framing, referendum, Catalonia. 


\section{Introducción}

La convocatoria de una "consulta sobre el futuro político de Cataluña" para el día 9 de noviembre de 2014, mediante el Decreto 129/2014, de 27 de septiembre (Parlament, 2014b), por parte del presidente de la Generalitat de Cataluña, Artur Mas, estuvo precedida y seguida por una intensa actividad institucional, social y cultural, que tuvo un importante reflejo en los medios de comunicación. Esta investigación estudia la representación mediática de los acontecimientos directamente vinculados con el referéndum propuesto, desde el ámbito teórico y metodológico del framing o enmarcado informativo.

\subsection{Del Estatut autonómico a la consulta sobre la independencia}

Según establece el Decreto 129/2014 de Presidencia de la Generalitat de Cataluña, esta convocatoria (mediática e institucionalmente popularizada como 9-N), respondía a la Resolución 5/X de 23 enero de 2013 por la que el Parlamento catalán "expresó la voluntad política de iniciar el proceso para que los ciudadanos de Cataluña pudieran ejercer el derecho a decidir el futuro político de Cataluña, atendiendo a los principios de legitimidad democrática, pluralismo y legalidad" (Parlament, 2014b).

A nivel legal esta iniciativa afirmaba ampararse en la Ley 10/2014 de la Generalitat de Cataluña, de 26 de septiembre, de consultas populares no refrendarias y de otras formas de participación ciudadana, en cuyo título II se establece que las autoridades competentes de la Generalitat podrán llamar a los ciudadanos para que manifiesten su opinión mediante votación sobre una determinada actuación, decisión o política pública de su ámbito competencial (Parlament, 2014c).

A pesar de que el Decreto de convocatoria afirmaba que la Generalitat de Cataluña tiene atribuida la competencia exclusiva en materia de consultas populares en los términos que establece el artículo 122 del Estatuto de Autonomía, tanto la Ley 10/2014 de consultas como este mismo Decreto fueron suspendidos unos días más tarde, el 29 de septiembre de 2014 por el Tribunal Constitucional (TC, 2014), sólo seis horas después de que el Gobierno de España los impugnara (El Mundo, 30 de septiembre).

El 6 de octubre de 2014 el portavoz de la Generalitat, Francesc Homs, fijaba en el día 15 de octubre la "fecha tope" para decidir si se convocaba la consulta, debido a los plazos legales marcados por la Ley 10/2014 (El Periódico, 7 de octubre). Finalmente, el 13 de octubre el presidente de Cataluña, Artur Mas, informaba a los partidos políticos que apoyaban el referéndum de que no podría celebrarse.

La noticia era publicada al día siguiente por toda la prensa española, en sus portadas por ejemplo: "Mas descarta la consulta y propone a cambio un "proceso participativo" (El País), "La Generalitat reconoce ya que el 9-N no habrá referéndum soberanista” (El Mundo), "Mas descarta la consulta prevista y propone un 9-N alternativo" (La Vanguardia), "La Ley doblega a Artur Mas. Renuncia a la consulta del 9-N” ( $A B C$ ), “Adios a la consulta” (El Periódico), “Últim intent. El govern manté que 
hi haurà urnes el 9-N i Mas explicarà avui un pla alternatiu a la consulta per poder votar" (El Punt Avui), "La Ley vence a Mas. Suspende la consulta del 9-N tras una tensa reunión con sus socios soberanistas" (La Razón), "Mas renuncia a la consulta del 9-N" ( La Voz de Galicia) o "Mas da marcha atrás y anuncia a sus socios que no será posible celebrar la consulta del 9-N" (Heraldo de Aragón).

Se ha afirmado por parte de diversos autores y representantes políticos que el punto de inflexión entre la reivindicación de mayores competencias autonómicas y una decidida reivindicación soberanista se produjo con la sentencia del Tribunal Constitucional de 9 de julio de 2011, por la que se anulaban catorce artículos del nuevo Estatut catalán, y se dejaba sin eficacia jurídica el término "nación” de su preámbulo. Así, Líndez (2013: 114) asegura que "el inicio del movimiento social secesionista se produce cuando el Estatut aprobado por los representantes elegidos por la ciudadanía catalana es recortado por un pacto con el gobierno central. Se inicia cuando se produce la primera decepción y frustración de las expectativas de un mayor nivel de autogobierno".

También Daniel Verdú en un reportaje publicado en el diario El País (22 de julio de 2014) considera que el Estatut es el "punto de origen de este fenómeno", pues si bien "fue una norma llamada desde su redacción a mejorar las relaciones entre Cataluña y el resto de España, a través de un mayor autogobierno (...) terminó generando el efecto contrario”. Desde una parte concreta del espectro político por ejemplo el secretario de Organización del PSOE, César Luena, se ha datado el origen de la frustración en Cataluña en el recurso del PP contra el Estatuto catalán de 2006 (Europa Press, 2014). Este nuevo Estatut había sido aprobado por el Parlamento de Cataluña en septiembre de 2005, y apoyado en el referéndum del 18 de junio de 2006 por el 73,9\% de los votantes, con una participación del 49,41\% del censo electoral.

Sin embargo, se han realizado otras argumentaciones explicativas en diferentes sentidos, apuntadas aquí a nivel meramente ilustrativo, siguiendo de nuevo a Líndez (2013: 89), basadas por ejemplo en el "expolio fiscal", el desequilibrio en la balanza fiscal entre lo que Cataluña aporta y lo que recibe del Estado en un momento de crisis económica, la política educativa del Gobierno central o las discrepancias sobre los símbolos catalanes.

Tras esta sentencia del Tribunal Constitucional en 2012 se produjeron una serie de grandes manifestaciones soberanistas. El 27 de septiembre de este año, el Parlamento de Cataluña aprobaba realizar un referéndum, con 84 votos a favor (CIU, ERC, ICV, SI), 21 en contra (PP y Ciutadans) y la abstención de los 25 diputados del PSC. Según la resolución, "el Parlamento de Cataluña constata la necesidad de que el pueblo de Cataluña pueda determinar libre y democráticamente su futuro colectivo, e insta al gobierno a hacer una consulta prioritariamente en la próxima legislatura" (Parlament de Catalunya, 2012: 3). Dos meses más tarde, el 25 de noviembre de 2012, se celebraron elecciones autonómicas anticipadas con la mayor participación de su historia (el 67,76\%), siendo de nuevo CIU la fuerza política más votada, si bien perdió 12 escaños, seguida por Esquerra Republicana, que duplicó sus votos y sus escaños (Gencat, 2014). 
Otros dos meses después, el 23 de enero de 2013, el Parlamento de Cataluña aprobó con 85 votos a favor, 41 en contra y 2 abstenciones la "Declaración de soberanía y del derecho a decidir del pueblo de Cataluña", donde se afirmaba que "el pueblo de Cataluña tiene, por razones de legitimidad democrática, carácter de sujeto político y jurídico soberano”, y se acordaba "iniciar el proceso para hacer efectivo el ejercicio del derecho a decidir para que las ciudadanas y los ciudadanos de Cataluña puedan decidir su futuro político colectivo” (Parlament, 2013). El 11 de septiembre de 2013, fiesta oficial de Cataluña, "Diada de Catalunya”, la Asamblea Nacional Catalana organizó una cadena humana de unos 400 km a lo largo de Cataluña, denominada "Vía Catalana hacia la Independencia” (Wikipedia, 2014).

El 16 de enero de 2014 el Parlamento de Cataluña acordaba la Resolución 479/X por la que se solicitaba al Congreso de los Diputados la delegación de competencias en la Generalitat de Cataluña "para autorizar, convocar y celebrar un referéndum consultivo para que los catalanes se pronuncien sobre el futuro político colectivo de Cataluña, en los términos que se acuerden con el Gobierno del Estado" (Parlament, 2014a: 5). Sin embargo, el Pleno del Congreso de los Diputados rechazó esta delegación de competencias el 8 de abril de 2014, por 299 votos en contra, 47 votos a favor y 1 abstención (Cortes Generales, 2014).

En 2014 de nuevo un 11 de septiembre fue escenario de una gran manifestación, en esta ocasión celebrada en la Gran Vía y en la avenida Diagonal de Barcelona, bajo el lema «Ahora es la Hora, unidos por un país nuevo». El número de congregados varió según la fuente entre la cifra mínima de medio millón de personas calculada por la Delegación del Gobierno a un máximo de 1.800 .000 personas según la Guardia Urbana $(A B C, 12$ de septiembre). Una cifra intermedia fue la calculada por el responsable del Servicio de Estadística Aplicada de la Universidad Autónoma de Barcelona, Llorenç Badiella, de 900 mil participantes (La Vanguardia, 12 de septiembre).

Apenas una semana después, el 19 de septiembre, el Parlamento de Cataluña aprobaba la ley de consultas con 106 votos a favor (CiU, ERC, PSC, ICV y CUP ) y 28 en contra (PP, Ciutadans) (La Vanguardia, 20 de septiembre de 2014), que entró en vigor mediante un decreto de Presidencia de la Generalitat el 27 de septiembre y quedó suspendida por el Tribunal Constitucional el día 29 de septiembre, como se ha descrito.

\subsection{La teoría del framing o de los marcos informativos}

La investigación en comunicación desde el marco teórico y metodológico del framing o enmarcado ha alcanzado un destacado desarrollo internacional, como muestra la revisión realizada por Weaver (2007) para el periodo 2001-2005, en la que encontró 165 investigaciones sobre framing, frente a 43 sobre agenda-setting y 25 sobre priming. Scheufele e Iyengar (2012: 2) afirman que hoy día cada ejemplar de las principales revistas científicas publican al menos un artículo sobre frames y efectos del framing. 
En España, según Vicente y López (2009: 27-29), "la comunidad científica que maneja los principios teóricos y prácticos del framing sigue siendo limitada" debido a la inexistencia de grandes equipos de investigación, la carencia de coordinación entre centros o a la escasa tradición investigadora en el campo de la comunicación, si bien destacan la producción de la Universidad de Navarra, la Pública de Salamanca y las de Madrid, citando expresamente al grupo dirigido por Juan José Igartua como "el más prolífico de la última década", en su línea de investigación del framing aplicado a la inmigración.

La definición de este concepto suele referirse a la obra de Erving Goffman "Frame Analysis. An Essay on the Organization of Experience" (1974), donde señala que "la definición de una situación (...) se construye de acuerdo con los principios de organización que gobiernan los hechos -al menos los sociales- y nuestra implicación subjetiva en ellos” (Goffman, 1974: 10). Para referirse a este concepto de frame emplea diferentes términos, como los de "definición de la situación" (Ibíd., 1), "perspectiva", "punto de vista” o "foco" (Ibíd., 8).

La construcción de los marcos informativos se produce, en opinión de Dader (1990: 128) como resultado de una "cooperación del equipo de actuantes" para producir "una versión convencional e industrialmente rentable. Tal cooperación contribuirá por fuerza a una reducción de la realidad en un conocimiento social estrecho", lo que lleva a concluir a este autor que una hipotética supresión de la institución periodística podría suponer "el inicio de un nuevo tipo de interacciones con la realidad", lo que supondría "una apertura mental incalculable".

Entman (1993: 52) ofrece la definición quizás más extendida de marco: "Enmarcar (to frame) es seleccionar algunos aspectos de una realidad percibida y hacerlos más destacados en un texto comunicativo, de tal forma que promueva una definición particular del problema, una interpretación de las causas, una evaluación moral, y/o una recomendación de tratamiento para el asunto descrito". Sin embargo, Scheufele e Iyengar (2002: 10) entienden que esta "omnicomprensiva" definición ha impulsado una vaguedad conceptual sobre el concepto de framing en numerosos estudios posteriores. Estos autores especifican que el enmarcado "se refiere a diferentes modos de presentación de una misma pieza de información" (Ibíd.,5), por lo que instan a los investigadores a considerar los marcos como variaciones en el modo de presentar un mismo mensaje, más que como manipulaciones en su naturaleza informativa o persuasiva.

Por su parte, Scheufele (1999) discrimina entre marcos de los medios y marcos de los individuos. A estos segundos se refiere Lakoff (2007: 17) al asegurar que los marcos son "estructuras mentales que conforman nuestro modo de ver el mundo". Pan y Kosicki (1993: 55) entienden el discurso informativo como un proceso que implica a fuentes, periodistas y audiencias "operando en el universo de la cultura compartida”. La concepción originaria de Goffman (1974: 24) ya indicaba que la elección de un marco determinado "proporciona una forma de describir el hecho al que se le aplica".

De modo complementario los marcos mediáticos funcionarían como rutinas de trabajo para los periodistas, ya que proveerían de una plantilla que guía a los periodistas para recopilar los elementos de la historia en una noticia (Gamson,1989; 
Pan \& Kosicki,1993). También Neuman et al. (1992: 60) indican que los marcos informativos son "herramientas conceptuales en las que se basan los medios y los individuos para transmitir, interpretar y evaluar información”. Esta investigación se centra en la descripción de los marcos mediáticos publicados en prensa escrita, pero teniendo en cuenta su íntima relación de doble sentido con los marcos de los individuos o estructuras mentales.

Pan y Kosicki (1993) señalan que el análisis del proceso de enmarcado evalúa diferentes dimensiones con las que se puede operar empíricamente, citando entre ellas las estructuras retóricas, temáticas, textuales y sintácticas (Ibíd., 55). El equipo de investigación del Observatorio de Contenidos Audiovisuales de la Universidad de Salamanca (OCA-USAL) propone una definición de frame, que a la vez que resume la evolución teórica de este concepto ofrece una metodología práctica para ser aplicada a investigaciones concretas:

Un encuadre noticioso remite al ángulo, enfoque, perspectiva o tratamiento de una información que se manifiesta en la elección, énfasis o importancia atribuida a diferentes elementos y, en particular, en la forma cómo covarían dichos elementos más o menos enfatizados en un texto. Mediante el análisis de la covariación o correlación entre el énfasis o importancia atribuida a cada elemento en los textos informativos es posible extraer los encuadres noticiosos como índices que permiten calificar o cualificar un mensaje individual o un sistema de mensajes (un conjunto o una muestra amplia de noticias). De este modo, en una misma noticia es posible identificar más de un encuadre, aunque uno de ellos resulte dominante. Por tanto, las palabras y expresiones clave relacionadas con los lugares geográficos, espacios físicos y temporales, actores protagonistas y acciones realizadas por / sobre ellos, atributos asociados al objeto de estudio son indicadores manifiestos a partir de los cuales se puede reconstruir la estructura latente y las dimensiones subyacentes del tratamiento informativo. (Igartua, Muñiz \& Cheng, 2005: 158)

La presente investigación adapta esta aproximación metodológica a nuestro concreto objeto de estudio, la consulta del 9$\mathrm{N}$, y comparte el interés de estos autores por conocer los "actores protagonistas y acciones realizadas por / sobre ellos" como indicadores del tratamiento informativo. Además, una importante función de los marcos, estudiada en este trabajo, sería la de aportar una evaluación a la información que transportan (Entman, 1993; Neuman et al., 1992; Tábara y Costejá, 2001).

Las fuentes informativas también han centrado la atención investigadora, remontándonos a autores clásicos como Laswell en su conocida frase de 1948: “Quién dice qué, en qué canal, a quién y con qué efecto”. De un modo más ceñido a nuestro instrumento de análisis, el papel de las fuentes en la conformación de los marcos ha sido descrito por diversos autores. Para Luhman (2000) los esquemas o scripts son estructuras que responden a los estímulos provenientes de determinadas fuentes, mientras que Porto (2002:2) se refiere como "esponsor" al promotor de "marcos interpretativos", que ofrece una interpretación determinada de un asunto político. También Entman (1993) considera a las fuentes de información como uno de los elementos mediante los que se manifiestan los marcos en el texto. 


\subsection{Objetivos}

1. Contribuir al desarrollo metodológico de la teoría del framing o enmarcado informativo, partiendo de un enfoque deductivo desde una aproximación prioritariamente cuantitativa, mediante el uso de un instrumental estadístico apropiado.

2. Describir los componentes de los marcos mediáticos de mayor relevancia formal que han operado en la información publicada de la consulta sobre la soberanía de Cataluña planteada para el 9 de noviembre de 2014.

3. Observar las asociaciones estadísticamente significativas entre los componentes de los marcos mediáticos y describir sus implicaciones informativas.

\subsection{Hipótesis}

Los medios escritos catalanes y madrileños realizaron un diferente tratamiento informativo de la consulta soberanista del 9 de noviembre de 2014.

\subsection{Preguntas de investigación}

Tomando como fundamento de nuestra investigación la línea teórica descrita en el apartado anterior, nos planteamos contestar a las siguientes preguntas de investigación en relación con nuestro objeto de estudio:

1. ¿ ¿Cuáles son las fuentes que han suministrado la información de partida de las piezas publicadas?

2. ¿ ¿Cuáles son los principales actores y las principales acciones que describe la prensa?

3. ¿ ¿Existen diferencias significativas entre el modo de evaluar las acciones realizadas por cada sujeto en función de la procedencia geográfica de la prensa española?

4. ¿Cómo se relacionan estos componentes en la composición del texto publicado?

5. ¿ ¿Utilizaron diferentes o similares marcos informativos los medios madrileños y catalanes sobre la consulta del 9-N?

\section{Metodología}

\subsection{Diseño y muestra}

Con el objeto de contestar a estas preguntas de investigación se realizó un estudio cuantitativo, llevándose a cabo un análisis de contenido de una selección de informaciones publicadas en la portada de la prensa escrita. Se eligieron los tres 
periódicos impresos de información general de Madrid (El País, El Mundo, ABC) y los tres de Cataluña (La Vanguardia, El Periódico, El Punt Avui) con mayor audiencia según el Estudio General de Medios más reciente (AIMC, 2014).

Cuadro 1. Audiencia de los periódicos analizados

\begin{tabular}{|c|c|}
\hline Medio & Audiencia \\
\hline El País & 1.685 .000 \\
\hline El Mundo & 1.032 .000 \\
\hline La Vanguardia & 690.000 \\
\hline El Periódico & 566.000 \\
\hline ABC & 517.000 \\
\hline El Punt Avui & 151.000 \\
\hline
\end{tabular}

Fuente: AIMC (2014)

Se recopilaron aquellas informaciones aparecidas en las portadas de los periódicos citados, publicadas entre el 20 de septiembre y el 3 de octubre de 2014, que contuviesen en su titular, o subsidiariamente en su subtítulo, las palabras "consulta" o "referéndum", o hiciesen referencia directa a estos asuntos. Se analizó la totalidad de la población de estudio, que resultó en un número de 96 informaciones publicadas.

El estudio se acotó a un intervalo temporal de dos semanas definido por eventos política e informativamente significativos, al corresponderse el primer día de estudio, el 20 de septiembre de 2014, con la publicación en los periódicos de la aprobación por parte del Parlamento catalán de la Ley de Consultas, y el último día de estudio, el 3 de octubre de 2014, con la información en prensa sobre la presentación de un recurso por parte de la Generalitat de Cataluña contra la decisión del Tribunal Constitucional de suspender la Ley de Consultas y la convocatoria de la consulta sobre la independencia de Cataluña para el 9 de noviembre.

A la hora de codificar la información se tuvo en cuenta sólo el titular principal de la información, y tan sólo cuando éste era difícil de codificar objetivamente por resultar una metáfora, un juego de palabras o una interpretación, se acudió a uno o varios subtítulos para interpretar el titular. Esta elección tuvo como objeto centrar la investigación en el marco principal o dominante que cada periódico destacó cada día. Los titulares son considerados por Pan y Kosicki (1993: 59) como la señal más destacada "para activar determinados conceptos semánticamente relacionados en las mentes de los lectores". Una decisión alternativa hubiera sido haber codificado tanto el título como los subtítulos como unidades de análisis diferentes, lo que hubiera podido mostrar cómo una noticia puede contener más de un marco informativo (Igartua, Muñiz \& Cheng, 2005). Frente a esta ventaja hubiéramos tomado en cuenta marcos secundarios a los que cada periódico otorga menor importancia. Por tanto, la opción elegida aportaba homogeneidad frente a variedad en los marcos detectados. 


\subsection{Codificación de la muestra}

Para codificar cada una de las unidades de análisis, se elaboró un libro de códigos en el que se determinaron las variables que serían analizadas, en los siguientes apartados:

a. Datos de identificación (número de unidad de análisis, fecha de publicación, texto del titular y texto del subtítulo).

b. Aspectos formales (orden de prioridad del titular, acompañamiento gráfico, aparición en portada, publicación en página impar). Se elaboró un índice de importancia propio, tomando como referentes los utilizados por Igartúa (2006) y Budd (1964 citado en Gutiérrez, 2001). Este índice describió la importancia de cada información como Mínima (0 puntos), Baja (1 puntos), Media (2 puntos), Alta (3 puntos) o Máxima (4 puntos), como resultado de sumar uno o cero puntos según los cuatro subapartados siguientes: 1. Aparición en portada: Sí (1), No (0); 2. Página: Par (0), Impar (1); 3. Acompañamiento gráfico: Sí (1), No (0); y 4. Orden de prioridad del titular: Primera o segunda noticia más destacada en portada (1), Tercera o siguientes noticias de portada (0). En nuestro caso dos de esas variables venían determinadas por el diseño de la investigación: todas las unidades de análisis aparecían en portada y por tanto en página impar.

c. Identificación de marcos informativos (fuente principal de la información, sujeto de la acción, campo semántico de la acción principal, hecho noticioso). Con esta última variable ("Hecho noticioso") se trató de mostrar cuál era, en esencia, el acontecimiento descrito, al registrar la combinación de actor protagonista (sujeto) y acontecimiento descrito (predicado) de cada unidad de análisis, continuando la línea de investigaciones anteriores (Ballesteros, 2012 y 2014).

e. Carácter evaluativo del acontecimiento principal. Se refiere al carácter evaluativo del suceso o hecho informativo para la sociedad, grupo o individuo afectado por la acción descrita. Se codificó como "carácter negativo", "carácter neutro o ambiguo" o "carácter positivo".

Todos estos atributos de la noticia fueron tratados como variables en un análisis estadístico con el que se trató de analizar la correlación existente entre ellos en términos estadísticamente significativos, siguiendo la línea de investigación descrita por Igartua, Muñiz y Cheng (2005).

\subsection{Procedimiento y fiabilidad}

Esta muestra de informaciones fue codificada mediante el programa SPSS v.15.0, calculándose una serie de estadísticos descriptivos (p.e. medias, medianas, modas y desviaciones típicas) e inferenciales para la determinación de la asociación estadística y su significación entre variables (Tablas de Contingencia, prueba exacta, V de Cramer). De este modo, para todos los resultados expresados en porcentajes se comprobó que existía significación estadística, precisándose en cada caso si es para valores de p menores de 0,01, 0,05 y 0,001, o tan sólo tendencialmente significativos, p menor de 0,1. 
La codificación de los datos fue realizada por un único juez, y con posterioridad un segundo juez realizó un nuevo análisis sobre una muestra del 33\% de las unidades elegidas al azar (32 noticias), a fin de estimar la fiabilidad del proceso de codificación (intercoder reliability). Para dicha revisión se contó con la ayuda de la doctora ingeniera industrial Beatriz de la Fuente Casado. El valor medio de acuerdo arrojado en las 8 variables que se tuvieron en cuenta (Kappa) fue de 0,82, un dato que señala una fiabilidad aceptable para el análisis realizado sobre las informaciones recogidas (Igartua, 2006; Neuendorf, 2002).

\section{Resultados}

En los catorce días sometidos a estudio los periódicos publicaron un total de 96 artículos en portada referidos a la consulta del 9-N, lo que supone la publicación de 6,9 noticias diarias. Este primer dato muestra la alta relevancia ofrecida por la prensa española a este acontecimiento informativo, pues cada uno de los seis periódicos publicó, de media, más de una noticia en portada cada día. Los diarios madrileños publicaron 42 informaciones (43,75\%) frente las 54 que publicaron los catalanes $(56,35 \%)$.

En su conjunto los medios madrileños informaron sobre la consulta en su portada todos los días de estudio, si bien $A B C$ no lo hizo cuatro de los catorce días (del 24 al 27 de septiembre), El Mundo tres días (21 y 25 de septiembre, y 1 de octubre) y El País sólo dejó de informar en portada sobre este asunto el día 27 de septiembre. Las noticias que ocuparon la primera página estas fechas fueron de gran calado informativo, como la dimisión del ministro de Justicia, Alberto Ruiz-Gallardón ( $A B C, 24$ de septiembre), la detención del presunto pederasta de Madrid ( $A B C$ y El Mundo, 25 de septiembre), la muerte de un misionero español por Ébola ( $A B C$, 26 de septiembre) o la comparecencia ante el Parlamento catalán del expresidente de la Generalitat, Jordi Pujol, acusado de fraude fiscal (ABCy El País, 27 de septiembre).

Los medios catalanes también publicaron noticias sobre el tema en portada cada día de estudio, y de modo individual no lo hicieron incluso menos días. La Vanguardia informó todos los días analizados. El Punt Avui solo dejó de hacerlo un día, el 24 de septiembre, en que publicó la dimisión de Gallardón. Hay que hacer constar que este periódico reprodujo a diario en su primera página una infografía bajo la cabecera que representaba una urna con el lema “9N" en su parte frontal y dos papeletas introduciéndose con un "Sí” en cada una de ellas. Junto a la urna se representó una cuenta atrás en la que cada día se informaba de los días que faltaban para la fecha de la consulta; por ejemplo el día citado: "Falten 46 díes”. Además, El Punt Avui recogió a diario una serie de noticias bajo un cintillo común denominado “\#Catalunyallibertat”, con el lema "Vol lliure en llibertat". Todo ello con los colores amarillo y rojo de la bandera catalana. Finalmente, El Periódico tan sólo relegó este tema de su primera página los días 24 y 25 de septiembre, en que informó sobre la dimisión del ministro de Justicia, las previsiones económicas del Banco de España y el final de las fiestas de Barcelona.

Además, las informaciones sobre el 9-N recibieron un relevante tratamiento formal, pues la importancia media de las noticias publicadas fue del 3,44 ( $D T=0,6)$, lo que supone una importancia entre "Alta” y “Máxima”, en un rango que oscilaba 
entre "Mínima" (0) y "Máxima" (4). En esta línea una mayoría de artículos recibieron un tratamiento de máxima importancia (4), valor en el que se situó la moda estadística. La mitad exacta de las noticias fueron de máxima importancia, mientras que un 43,8\% lo fueron de alta importancia. Tan sólo un 6,3\% fueron de importancia media, y de ellas la mitad complementaban a la noticia principal del día sobre el mismo tema. El índice de importancia media de los diarios madrileños ( $\mathrm{M}=3,46$, DT = 0,58) resultó similar al de los diarios catalanes $(M=3,50, D T=0,64)$, pues al realizar la prueba t de Student para muestras independientes se comprobó que la diferencia entre las medias de ambos grupos no resulta significativa $(t(94)=-1,14, p>0,05)$.

En la mitad de las piezas analizadas (51\%) la fuente de información fueron las instituciones catalanas. A distancia se situaron las instituciones españolas (15,6\%) y el propio periodista o fuente indeterminada (15,6\%). Otras fuentes empleadas fueron los políticos españoles $(6,3 \%)$ y catalanes $(2,1 \%)$, juristas $(3,1 \%)$ y ciudadanos $(2,3 \%)$. No se detectaron diferencias significativas entre las fuentes utilizadas por medios catalanes o madrileños.

Las instituciones catalanas sobresalieron como sujeto protagonista de los hechos descritos con un $62,5 \%$ de los casos, seguidas por las instituciones españolas (17,7\%). Otros protagonistas destacados fueron los políticos, con un 13,6\%, repartidos entre españoles $(7,3 \%)$, catalanes $(4,2 \%)$ y la mezcla de ambos $(2,1 \%)$. Los ciudadanos también fueron protagonistas en un 6,3\% de las ocasiones. De este modo, los dos sujetos institucionales protagonizaron más del $80 \%$ de las noticias, lo que sumado al protagonismo de cargos y partidos políticos, sumó un total del 93,8\%. Se trató por tanto de una información intensamente protagonizada por políticos. La prueba exacta de Fischer tampoco mostró diferencias significativas entre los sujetos empleados por los periódicos catalanes y los madrileños.

Los protagonistas de la información fueron personas físicas el 42,7\% de las ocasiones, mientras que el 57,2\% lo fueron los sujetos jurídicos anteriormente citados. De entre los sujetos físicos que protagonizaron noticias destacó el presidente de la Generalitat, Artur Mas, con el 80,5\% de los casos, seguido a gran distancia por el secretario general del Partido Socialista, Pedro Sánchez, con el 9,8\%, el presidente del Gobierno, Mariano Rajoy, con el 4,9\%, el lehendakari, Iñigo Urkullu, con el 2,3\%, y el alcalde de Barcelona, Xavier Trías, con otro 2,3\%. Si tomamos la muestra global, incluyendo tanto a personas físicas como jurídicas, Artur Mas fue el protagonista del 34,4\% de las noticias; Pedro Sánchez , secretario general del PSOE, protagonizó el 4,2\% y Mariano Rajoy el 2,1\%.

La principal acción realizada por estos sujetos fue de tipo político $(79,2 \%)$, seguida por acciones que implicaban un delito o un acto ilegítimo (18,8\%). Se calculó la tabla de contingencia entre las variables "Accion descrita" y "Comunidad Autonóma” en que se publica el periódico, resultando una asociación significativa (Prueba exacta de Fisher $=22,14, \mathrm{p}<0,01, \mathrm{~V}$ $=0,479)$. Las acciones políticas fueron las principalmente descritas tanto por los periódicos madrileños $(57,1 \%)$ como por los catalanes (96,3\%), pero mientras que el $38,1 \%$ de los medios madrileños describieron acciones de tipo ilegal, los catalanes lo hicieron diez veces menos, en tan sólo el 3,7\% de los casos. 
Un ejemplo de este tipo de acciones cercanas al delito lo encontramos en titulares como "Mas desoye el aviso escocés y convocará el referéndum ilegal" (Unidad de Análisis número 1, $A B C, 20$ de septiembre de 2014), "Mas firma la ruptura de la legalidad" (UA 19, El Mundo 29 de septiembre) o "Mas desafía al Constitucional y crea la junta para votar el 9-N" (UA 42, El País, 3 de octubre).

Mediante la variable "Hecho noticioso" se describieron las posibles combinaciones de actores y acciones en cada noticia. Así se obtuvieron los seis "hechos noticiosos" o "sucesos informativos" más habituales relacionados con la consulta del 9$\mathrm{N}$, que totalizaron casi el 95\% de la muestra. La principal noticia de que informó la prensa escrita con respecto al 9-N, fue que una institución catalana realizara una acción de tipo político, con un 42,7\% del total. Este tipo de informaciones se reflejó en titulares como "Mas fija en el 9-N el inicio del cambio constitucional” (UA 66, El Periódico, 23 de septiembre) o "El pes de la llei. La llei de consultes és aprovada al Parlament amb un suport històric” (UA79, El Punt Avui, 20 de septiembre).

La siguiente noticia más habitual también tuvo como sujeto a las instituciones catalanas, pero en este caso realizando acciones de tipo ilegal o ilegítimo, como las citadas anteriormente, en un 17,7\% de ocasiones. De igual modo, los políticos catalanes fueron protagonistas de acciones de tipo ilegal en un 1,0\% de veces. Protagonizaron acciones de tipo político las instituciones españolas (17,7\%), los políticos catalanes (3,1\%), españoles $(7,3 \%$ y los ciudadanos (6,3\%) (Datos en cuadro 2).

Cuadro 2. Principales hechos noticiosos relacionados con el 9-N

\begin{tabular}{|c|c|c|c|}
\hline \multicolumn{2}{|c|}{ Hecho noticioso } & \multirow{2}{*}{ Frecuencia } & \multirow{2}{*}{ Porcentaje } \\
\hline Sujeto & Acción descrita & & \\
\hline Institución catalana & Política & 41 & 42,7 \\
\hline Institución catalana & Delito & 17 & 17,7 \\
\hline Institución española & Política & 17 & 17,7 \\
\hline Político español & Política & 7 & 7,3 \\
\hline Ciudadanos & Política & 6 & 6,3 \\
\hline \multirow[t]{2}{*}{ Político catalán } & Política & 3 & 3,1 \\
\hline & Resto & 5 & 5,2 \\
\hline \multicolumn{2}{|c|}{ Total } & 96 & 100 \\
\hline
\end{tabular}

Fuente: Elaboración propia

Se calculó una tabla de contingencia entre las variables sujeto y acción descrita, resultando una relación no significativa (Prueba exacta de Fisher $=14,73, p=0,121$ ). En este sentido, la prueba $V$ de Cramer desestimó una asociación entre ambas 
$(V=0,259, p=0,231)$, lo que implica que, en la muestra global, cada sujeto no estaba especialmente asociado a una acción concreta.

Segmentando el archivo en dos muestras según la procedencia geográfica del periódico se halló que mientras que los periódicos madrileños sí relacionaban significativamente el sujeto y la acción descrita, no lo hacían así los periódicos catalanes. En el caso de los diarios madrileños se calculó un valor de la prueba exacta de Fisher = 19,753 $(p<0,001, V=$ 0,456 ), y en el de los diarios catalanes una prueba exacta de Fisher $=6,7$ ( $p=0,331, V=0,388$ ). Estos datos indicaban que los medios de Madrid tendieron a representar a cada sujeto realizando una acción característica, mientras que en los medios de Barcelona no se pudo encontrar una acción asociada a cada sujeto.

Para profundizar en esta línea, se calculó una tabla de contingencia entre la variable "Hecho noticioso" (que cruzaba Sujeto y Acción) con la variable Comunidad Autónoma del medio. Se halló una relación significativamente estadística del mayor nivel posible entre ambas variables (Prueba exacta de Fisher $=33,946, p<0,001, V=0,589$ ), lo que demostraba que la elección del hecho noticioso estaba íntimamente relacionado con la procedencia geográfica del medio que lo publicaba. De este modo, los periódicos madrileños relacionaron mayoritariamente a las instituciones catalanas con acciones ilegales (38,1\%), y tan sólo en un 21,4\% con actividades políticas.

En cambio los periódicos catalanes relacionaron a las instituciones catalanas principalmente con actividades políticas (59,3\%), y en solo una ocasión (1,9\%) con actividades posiblemente delictivas. La única vez en que un periódico catalán informó de una institución catalana protagonizando una acción ilícita se trató de la unidad de análisis número 76, titulada "Los riesgos de la desobediencia. Informes jurídicos" (El Periódico, 1 de octubre), que se recodificó como "Las instituciones catalanas crearían riesgos a Cataluña en caso de desobedecer la ley, según informes jurídicos”.

En contraste, los medios catalanes nunca achacaron acciones ilegales a las instituciones políticas españolas, como tampoco lo hicieron los medios madrileños, e incluso relacionaron a los propios políticos catalanes con acciones ilegales en un 1,9\% de ocasiones, mientras que los medios madrileños no lo hicieron ninguna (Datos en Cuadro 3). 
Cuadro 3. Principales hechos noticiosos relacionados con el 9-N según CCAA del periódico

\begin{tabular}{|c|c|c|c|c|}
\hline \multicolumn{2}{|c|}{ Hecho noticioso } & \multirow{2}{*}{ Periódico madrileño } & \multirow{2}{*}{ Periódico catalán } & \multirow{2}{*}{ Tota } \\
\hline Sujeto & Acción descrita & & & \\
\hline Institución catalana & Política & 21,4 & 59,3 & 42,7 \\
\hline Institución catalana & Delito & 38,1 & 1,9 & 17,7 \\
\hline Institución española & Política & 21,4 & 14,8 & 17,7 \\
\hline Político español & Política & 9,5 & 5,6 & 7,3 \\
\hline Ciudadanos & Política & 2,4 & 9,3 & 6,3 \\
\hline Político catalán & Política & 2,4 & 3,7 & 3,1 \\
\hline \multirow[t]{2}{*}{ Político catalán } & Delito & 0 & 1,9 & 1,0 \\
\hline & Resto & 4,8 & 3,5 & 4,2 \\
\hline \multicolumn{2}{|c|}{ Total } & 100 & 100 & 100 \\
\hline
\end{tabular}

Nota: $\mathrm{N}=96$. Los valores de las celdas representan porcentajes. La asociación es significativa a nivel ${ }^{* * *} \mathrm{p}<, 001$. Fuente: Elaboración propia

La evaluación del acontecimiento principal resultó negativa en un 33,3\% de ocasiones, neutra en el 44,8\% y positiva en el 21,9\%. La prueba t de Student para muestras independientes señaló diferencias significativas para la evaluación del acontecimiento principal realizada por medios catalanes y madrileños $(t(94)=-2,95 p<0,01)$. A continuación se calculó que la asociación entre las variables "Evaluación del acontecimiento principal” y "Comunidad Autonóma del periódico" resultaba estadísticamente significativa (Prueba exacta de Fisher $=16,30, p<0,001, V=0,412$ ).

Así, se halló que los diarios madrileños evaluaron las noticias sobre el 9-N mayoritariamente como negativas (54,8\%), frente a un $26,4 \%$ de noticias neutras y un $19 \%$ de noticias positivas. En cambio los diarios catalanes consideraron mayoritariamente neutras las noticias sobre el 9-N (59,3\%), positivas en un 24,1\% de ocasiones y negativas en el 16,7\%. También resultó estadísticamente significativa la asociación entre las variables "Hecho noticioso" y "Evaluación del acontecimiento principal" (Prueba exacta de Fisher $=56,034, p<0,001, V=0,564$ ). 
Cuadro 4. Evaluación de los principales hechos noticiosos relacionados con el 9-N

\begin{tabular}{|c|c|c|c|c|c|}
\hline \multicolumn{2}{|c|}{ Hecho noticioso } & \multicolumn{2}{c|}{ Evaluación del acontecimiento principal } & \multirow{2}{*}{ Total } \\
\hline Sujeto & Principal acción descrita & Negativa & Neutra o ambigua & Positiva & \multirow{2}{*}{100} \\
\hline Institución catalana & Política & 12,2 & 58,5 & 29,3 & 100 \\
\hline Institución catalana & Delito & 100 & 0 & 0 & 100 \\
\hline Institución española & Política & 23,5 & 41,2 & 35,3 & 100 \\
\hline Político catalán & Política & 0 & 100 & 0 & 100 \\
\hline Ciudadanos & Política & 0 & 83,3 & 16,7 & 100 \\
\hline Político español & Política & 42,9 & 28,6 & 28,6 & 100 \\
\hline Político catalán & Delito & 100 & 0 & 0 & 100 \\
\hline
\end{tabular}

Nota: $\mathrm{N}=96$. Los valores de las celdas representan porcentajes. La asociación es significativa a nivel ${ }^{* * *} \mathrm{p}<, 001$. Fuente: Elaboración propia

Las acciones políticas de las instituciones catalanas fueron evaluadas mayoritariamente como neutras (58,5\%), positivas en un 29,3\% de ocasiones y negativas en sólo un 12,2\% (Datos en Cuadro 4). Estas cifras revelaron de nuevo todo su significado al segmentar la muestra y descubrir que los periódicos madrileños nunca evaluaron de modo positivo las acciones políticas de las instituciones catalanas, sino que lo hicieron de modo neutro $(66,7 \%)$ y negativo (33,3\%). Un ejemplo de acción política negativa protagonizada por una institución catalana lo encontramos en la unidad de análisis 21 "El Consejo de Estado dice que el Parlament no puede legislar sobre el referéndum” (El Mundo, 29 de septiembre).

Los medios catalanes también evaluaron de un modo neutro las acciones políticas de las instituciones catalanas (56,3\%), pero en contraste evaluaron en mayor número de ocasiones estas acciones como positivas (37,5\%) que como negativas (6,3\%) (Datos en Cuadros 5 y 6). El siguiente hecho noticioso más descrito, las acciones posiblemente ilegales de las instituciones catalanas, fue evaluado siempre de modo negativo. Este fue el principal suceso informativo para los periódicos madrileños, mientras que la prensa catalana sólo describió acciones ilegales por parte de las instituciones catalanas una vez y en ninguna ocasión se las achacó a las instituciones españolas.

La evaluación neutra para la muestra global también fue mayoritaria en el caso de las acciones políticas de las instituciones españolas (41,2\%), seguida por una evaluación positiva en el 35,3\% de los casos y negativa en el $23,5 \%$. Resulta ilustrativo comprobar en los Cuadros 5 y 6 que en los diarios madrileños las acciones políticas de las instituciones españolas son evaluadas como positivas (66,7\%), mientras que los diarios catalanes nunca las evaluaron positivamente, y sí 
negativamente en un 37,5\%. Todas estas evaluaciones negativas se publicaron en un único medio, el diario El Punt Avui, por lo que tanto La Vanguardia como El Periódico evaluaron siempre de modo neutro a las instituciones españolas.

Las repercusiones económicas actuales o futuras de la propuesta de la consulta del 9-N no fueron tratadas en portada por la prensa catalana, y apenas por la madrileña, ya que sólo apareció en dos ocasiones. Ambas veces la evaluación fue negativa, tanto en la unidad de análisis 9 ("Fitch amenaza con rebajar la nota de la deuda catalana a 'bono basura", $A B C, 30$ de septiembre de 2014) como en la UA 23 ("Es improbable que los inversores compren la deuda de Cataluña. Fitch la pone en perspectiva negativa y amenaza con degradarla a 'bono basura”, El Mundo, 30 de septiembre de 2014).

Cuadro 5. Evaluación de los principales hechos noticiosos relacionados con el 9-N por parte de los medios madrileños

\begin{tabular}{|c|c|c|c|c|c|}
\hline \multicolumn{2}{|c|}{ Hecho noticioso } & \multicolumn{2}{c|}{ Evaluación del acontecimiento principal } & \multirow{2}{*}{ Total } \\
\hline Sujeto & Principal acción descrita & Negativa & Neutra o ambigua & Positiva & 100 \\
\hline Institución catalana & Política & 33,3 & 66,7 & 0 & 100 \\
\hline Institución catalana & Delito & 100 & 0 & 0 & 100 \\
\hline Institución española & Política & 11,1 & 22,2 & 66,7 & 100 \\
\hline Político catalán & Política & 0 & 100 & 0 & 100 \\
\hline Ciudadanos & Política & 0 & 100 & 0 & 100 \\
\hline Político español & Política & 25 & 25 & 50 & 100 \\
\hline Institución catalana & Economía & 100 & 0 & 0 & 100 \\
\hline
\end{tabular}

Nota: $\mathrm{N}=42$. Los valores de las celdas representan porcentajes. La asociación es significativa a nivel ${ }^{* * *} \mathrm{p}<, 001$. Fuente: Elaboración propia 
Cuadro 6. Evaluación de los principales hechos noticiosos relacionados con el 9-N por parte de los medios catalanes

\begin{tabular}{|c|c|c|c|c|c|}
\hline \multicolumn{2}{|c|}{ Hecho noticioso } & \multicolumn{3}{|c|}{ Evaluación del acontecimiento principal } & \multirow{2}{*}{ Tota } \\
\hline Sujeto & Principal acción descrita & Negativa & Neutra o ambigua & Positiva & \\
\hline Institución catalana & Política & 6,3 & 56,3 & 37,5 & 100 \\
\hline Institución catalana & Delito & 100 & 0 & 0 & 100 \\
\hline Institución española & Política & 37,5 & 62,5 & 0 & 100 \\
\hline Político catalán & Política & 0 & 100 & 0 & 100 \\
\hline Ciudadanos & Política & 0 & 80 & 20 & 100 \\
\hline Político español & Política & 66,7 & 33,3 & 0 & 100 \\
\hline \multirow[t]{2}{*}{ Político catalán } & Delito & 100 & 0 & 0 & 100 \\
\hline & & 16,7 & 59,3 & 24,1 & \\
\hline
\end{tabular}

Nota: $\mathrm{N}=54$. Los valores de las celdas representan porcentajes. La asociación es significativa a nivel $* \mathrm{p}<, 05$. Fuente: Elaboración propia

Evaluaciones positivas de las acciones políticas de las instituciones españolas en los periódicos madrileños se encontraron, por ejemplo, en unidades de análisis como la número 5 ( $A B C$, 28 de septiembre de 2014), titulada "El Gobierno activa el Estado de Derecho para tumbar el 9-N en Cataluña”. La evaluación positiva de la actividad del Gobierno procede de relacionarla con la lógica del Estado de Derecho como reacción al hecho descrito en el subtítulo de esta noticia: "Mas consuma su rebelión contra España”. Para reforzar la carga positiva de esta acción, la imagen a toda portada estaba ocupada por el lema a grandes tipos: "La hora de la ley. Constitución Española”.

Otra evaluación positiva de las instituciones españolas se ilustra en la unidad de análisis 38 (El País, 30 de septiembre de 2014), con el título: "El Constitucional suspende la consulta y desmonta el plan de Mas”, que de nuevo reafirma su carga favorable en toda su plenitud al completarse con el subtítulo "Respuesta al desafío secesionista".

Por el contrario, encontramos un ejemplo de cómo los diarios madrileños evaluaron negativamente las acciones de las instituciones españolas en la unidad de análisis 24 (El Mundo, 2 de octubre de 2014 "Mas avisa de que suspender el referéndum generará 'violencia”. Este titular fue recodificado como "Si las instituciones españolas suspenden el reférendum se generará violencia, según Mas”. Se le adjudicó una evaluación negativa a la acción política de las instituciones españolas de acuerdo con la fuente de esta información, el presidente catalán Artur Mas. Esta misma norma de codificación se siguió con toda la muestra analizada, respetando el sentido evaluativo que fuente y diario dan a sus informaciones, y evitando por tanto una segunda interpretación desde la subjetividad del codificador. 
Por su parte, podemos ilustrar algunas evaluaciones negativas de las acciones de las instituciones españolas en los diarios catalanes con la unidad de análisis número 80 (El Punt Avui, 21 de septiembre de 2014), titulada "Mala peça al TC" ${ }^{1}$, cuyo subtítulo rezaba "La influència partidista fa dubtar de la independència del tribunal que haurà de valorar la llei y el decret de consultes". En la misma línea podemos citar la UA 90, con el título "Van a sac", y los subtítulos "El TC admet a tràmit els recursos del govern de l'Estat i suspèn la llei de consultes i el decret” y “Mas: 'Ni pregunten ni diexen preguntar. Temen la resposta". En este caso se acusa a las instituciones españolas de "ir a saco", al suspender la ley de consultas y el decreto de convocatoria de la consulta del 9-N.

\section{Conclusiones}

Los periódicos españoles, tanto los radicados en Madrid como en Barcelona, han informado muy intensamente sobre la consulta del 9-N durante las dos semanas analizadas, de modo que todos los días de estudio aparecieron noticias en portada en un mínimo de tres diarios distintos, y hasta en ocho días lo hicieron en los seis medios analizados. Así, en 74 de las de 84 portadas del periodo de estudio aparecieron informaciones sobre la consulta.

Además, la relevancia formal con que se trató este asunto fue alta o máxima, ya que la mitad de las noticias analizadas fueron la principal información de portada. La mayor parte del resto de noticias (el 43,8\%) fueron la segunda noticia del día. La importancia que medios catalanes y madrileños dieron a estas informaciones fue similar.

En contestación a la primera pregunta de investigación, las principales fuentes de información fueron de tipo institucional y político, en tres de cada cuatro ocasiones. Por el contrario, las fuentes menos empleadas en este asunto fueron los ciudadanos, que apenas superaron el 2\%. Si la ley ha impedido ejercer el denominado "derecho a decidir" de los ciudadanos catalanes, al menos en los términos en que esta consulta se planteaba, parece que los medios madrileños y catalanes han postergado de los espacios informativos prioritarios el derecho de expresión de los ciudadanos.

En cuanto a la segunda pregunta de investigación, el protagonista principal de las informaciones sobre el 9-N, tanto en la prensa madrileña como catalana, fueron las instituciones catalanas, casi seis de cada diez veces. Le siguieron a mucha distancia las instituciones españolas, con menos de dos de cada diez. Los dos casos restantes se los repartieron entre políticos y ciudadanos, si bien los primeros lo hicieron en más del doble de ocasiones. Por tanto, se comprueba que la prensa escrita no sólo eligió a las fuentes institucionales y políticas para informar sobre este tema, sino que la información resultante estuvo protagonizada en más de un $90 \%$ por cargos políticos e institucionales. Los ciudadanos ni informan ni son protagonistas de la información sobre el 9-N en los espacios más relevantes.

\footnotetext{
1 “Mala peça al TC” (Tribunal Constitucional): literalmente "Mala pieza en el TC”, remite a la expresión "mala peça”, persona de mal comportamiento, así como a "tenir mala peça al teler”, encontrarse en una situación difícil, una enfermedad difícilmente curable, ir a mal fin (IEC, 2014).
} 
En contraste, se produjo una gran personalización de este asunto informativo en la figura del presidente de la Generalitat, Artur Mas, que protagonizó (con su propio nombre, a menudo sin citar el cargo) más del 34\% de las noticias. El líder del Partido Socialista apenas superó el 4\%, y el propio presidente del Gobierno se quedó en un 2,1\%. Esta personalización se centró en el presidente de la Generalitat de un modo incluso más intenso en los periódicos madrileños (38\% de protagonismo) que en los catalanes (22\%), que repartieron más este protagonismo con las instituciones. Si contamos sólo las personas físicas, y no las jurídicas, Artur Mas fue el protagonista del $80 \%$ de las noticias.

La principal acción que protagonizaron estos sujetos fue de tipo político, en casi un $80 \%$ de las veces, y de tipo ilegal en casi el 20\% restante. Sin embargo, en esta ocasión, sí se encontraron diferencias significativas entre las acciones descritas por los medios de una u otra Comunidad Autónoma. Así, los periódicos madrileños informaron no sólo de acciones políticas (en un mayoritario 57\%), sino también de otro tipo de acciones que eran o podrían ser consideradas ilegales, en un $38 \%$ de casos. En cambio los medios catalanes relacionaron la consulta casi siempre con acciones de tipo político (96\%), y en apenas un $4 \%$ la relacionaron con algún tipo de acto ilegal. Por tanto, la variable "Acción descrita" sirvió para detectar una importante diferencia en el uso de los marcos informativos, pues los diarios madrileños introdujeron un marco que definía una serie de acciones como ilegales.

La siguiente variable analizada mostró que los periódicos madrileños atribuyeron estas acciones ilegales siempre a las instituciones catalanas. De este modo, el principal hecho noticioso de que informaron los diarios madrileños fue la acción ilegal, presente o futura, de una institución catalana, en el 38\% de ocasiones. Los dos siguientes sucesos informativos de la prensa madrileña fueron las acciones políticas de instituciones españolas y catalanas, que sumaron cada una un $21 \%$. Se comprobó que la prensa madrileña asoció significativamente cada acción con un sujeto determinado, en la línea de anteriores investigaciones (Ballesteros, 2012 y 2014) que ya detectaron un reparto fijo de papeles (fixed role casting) en determinados sucesos informativos.

En cambio la prensa catalana informó como principal hecho noticioso de acciones políticas de las instituciones catalanas (59\%), seguidas por las acciones políticas de las instituciones españolas (15\%). El delito apenas fue relacionado con la consulta, y cuando lo fue, 1,9\% del total, lo hizo para relacionarlo con políticos catalanes. Sin embargo no asoció significativamente a cada actor con una acción característica.

La tercera pregunta de investigación sirvió para detectar diferencias significativas entre el modo de evaluar las acciones realizadas por cada sujeto en función de la procedencia geográfica de la prensa española. Así, la prensa madrileña evaluó el 9-N sobre todo de un forma negativa (55\%) y neutra (26\%), mientras que la prensa catalana lo hizo neutral (59\%) y positivamente $(24 \%)$.

En concreto fueron las instituciones y los políticos catalanes quienes protagonizaron los hechos noticiosos negativos y neutros descritos en los diarios madrileños, mientras que las instituciones y los políticos españoles eran relacionados muy 
mayoritariamente con asuntos informativos de carácter positivo. En cambio los diarios catalanes informaron de cómo las instituciones catalanas realizaban hechos noticiosos neutros o positivos, mientras que las instituciones y políticos españoles realizaban acciones neutras o negativas.

Por tanto se corroboró nuestra hipótesis de partida ya que, si bien el enmarcado informativo de la prensa escrita catalana y madrileña mostró ciertas coincidencias nucleares (en las fuentes y en los sujetos protagonistas), el tratamiento del 9-N por parte de cada grupo de medios utilizó de modo diferente ciertos componentes esenciales de los marcos como la evaluación, que resultó de signo contrario, y la interpretación por parte de los periódicos madrileños de que la Generalitat estaba incumpliendo o proponiendo incumplir la ley, que apenas fue descrita por los medios catalanes.

Mientras que los diarios de Madrid presentaron un proceso cargado de tintes negativos que proponía vulnerar la ley, los diarios de Barcelona relataron el normal funcionamiento político de las instituciones dentro de un Estado de Derecho, de un modo neutral cuando no positivo. Se comprobó que ambos grupos mediáticos tiñeron sus informaciones con un tinte nacionalista, de manera que los políticos y las instituciones que les resultan más próximos tienden a protagonizar acciones evaluadas de un modo más positivo, mientras que los políticos e instituciones más lejanos reciben un tratamiento informativo más negativo.

Podríamos concluir mediante un símil gráfico con que la prensa de cada Comunidad Autonóma actuó como negativo fotográfico de la prensa de la otra Comunidad. Si bien el parecido metafórico sería aun mayor con la imagen del yin y yang, donde una parte del dibujo es simétrica y de color opuesto a la otra (como ocurre en nuestro estudio con la evaluación y los hechos noticiosos), pero todavía comparte en su interior un punto nuclear del mismo color que su opuesto, como en nuestro caso ocurre con la coincidencia de fuentes y sujetos protagonistas. Más allá de límites territoriales, los periodistas parecen compartir un saber operativo profesional sobre quienes les proveen de información y quienes la protagonizan.

Por el contrario el debate sobre Cataluña está acompañado por opuestas representaciones informativas por parte de los medios de cada territorio. De modo simbólico, si las fronteras geográficas aún no separan naciones de derecho sí que parecen determinar naciones informativas de hecho, caracterizadas por aportar interpretaciones propias e incompatibles de los mismos procesos políticos.

Los efectos de la exposición a tan diferentes marcos mediáticos sobre los marcos mentales de los ciudadanos y su repercusión sobre la vida política, social y cultural podrá representar otra interesante línea de investigación futura. También corresponderá a posteriores investigaciones indagar las condiciones que hacen que se produzca o no el fenómeno detectado del reparto fijo de papeles en las informaciones de la prensa. 


\section{Referencias bibliográficas}

AIMC (2014): Resumen general de resultados EGM. Octubre de 2013 a mayo de 2014. http://www.aimc.es/spip.php?action=acceder_document\&arg=2590\&cle=220f931df0e4b91629bc5367091ff 4 bf 1 babalb9\&file=pdf\%2Fresumegm214.pdf [Consultado el 06/10/2014].

Alòs, J. S. (1995): “Técnicas de encuesta por muestreo”. http://www.aedemo.es/aedemo3/pdf/muestreo.pdf [Consultado el 20/10/2014].

Ballesteros, C. (2012): Los marcos informativos del cannabis en la prensa española: Aplicación de las teorías del framing y la agenda-setting. Saarbrücken: Editorial Académica Española.

Ballesteros, C. (2014): "El enmarcado informativo del cannabis: un estudio cuantitativo exploratorio desde la teoría del framing", Anuario Electrónico de Estudios en Comunicación Social "Disertaciones", n.7 (1), pp. 67-103. http://erevistas.saber.ula.ve/index.php/Disertaciones/ [Consultado el 07/09/2014].

Cortes Generales (2014): “Toma en consideración de proposiciones de ley: Del Parlamento de Cataluña, orgánica de delegación en la Generalidad de Cataluña de la competencia para autorizar, convocar y celebrar un referéndum sobre el futuro político de Cataluña", Diario de Sesiones del Congreso de los Diputados. http://www.congreso.es/public_oficiales/ L10/CONG/DS/PL/DSCD-10-PL-192.pdf [Consultado el 07/10/2014].

Dader, J. (1990): “Las provincias periodísticas de la realidad”, Comunicación y Sociedad, n. 3 (1-2), pp. 85-134.

Entman, R. (1993): "Framing: toward clarification of a fractured paradigm”, Journal of Communication, n. 43 (4), pp. 51-58.

Europa Press (2014): "Luena tacha a Rajoy de "comodón" por dar largas a su propuesta de reforma constitucional". http://www.europapress.es/nacional/noticia-consulta-luena-tacha-rajoy-comodon-dar-largas-propuesta-reformaconstitucional-20141001130052.html [Consultado el 07/10/2014].

Gamson, W. (1989): “News as framing: Comments on Graber”, American Behavioral Scientist, n. 33, pp. 157-161.

Gencat (2014): “Elecciones al Parlament de Catalunya. Resultados definitivos”. http://www.gencat.cat/governacio/resultats-parlament2012/09AU/DAU09999CM_L1.htm [Consultado el 28/09/2014].

Goffman, E. (1974): Frame Analysis. An Essay on the Organization of Experience. New York: Harper Colophon.

Gutiérrez, L. (2001): “Temas de interés para los periódicos de prestigio. El caso de El País (España) y El Espectador (Colombia)", Zer, n. 10, pp. 131-158. http://dspace.unav.es/ dspace/bitstream/10171/16976/1/ZER\%20100002.pdf [Consultado el $07 / 10 / 2007]$.

IEC (2014): “Peça”, Diccionari de la llengua catalana. Institut d'Estudis Catalans. http://dlc.iec.cat/results.asp?txtEntrada=pe\%E7a [Consultado el 22/10/2014].

Igartua, J. (2006): Métodos cuantitativos de investigación en comunicación. Barcelona: Bosch. 
Igartua, J., Muñiz, C. y Cheng, L. (2005): “La inmigración en la prensa española. Aportaciones empíricas y metodológicas desde la teoría del encuadre noticioso”, Migraciones, n. 17, pp. 143-181.

Lakoff, G. (ed. 2007): No pienses en un elefante. Lenguaje y debate político. Madrid: Editorial Complutense.

Líndez, M. C. (2013): “Movilización en la sociedad catalana: aparición y pervivencia”, Clivatge, n. 2, pp. 88-116. revistes.ub.edu/index.php/clivatge/article/download/10022/12891 [Consultado el 07/10/2014].

Luhmann, N. (2000): La realidad de los medios de masas. Barcelon:. Anthropos Editorial.

Neuendorf, K. A. (2002): The Content Analysis Guidebook. Thousand Oaks, CA: Sage Publications.

Pan, Z. \& Kosicki, G. (1993): “Framing analysis: An approach to news discourse”, Political Communication, n. 10 (1), pp. 55-75.

Parlament de Catalunya (2012): "Resolució de 27 de septiembre de 2012". http://www.ara.cat/politica/resolucio-CiU-ERCautodeterminacio-Parlament_ARAFIL20120926_0001.pdf [Consultado el 07/10/2014].

Parlament de Catalunya (2013): "Resolució de 13 de enero de 2013. Declaración de Soberanía y del derecho a decidir del pueblo de Cataluña”. http://www.parlament.cat/web/actualitat/noticies?p_id=129656021 [Consultado el 07/10/2014].

Parlament de Catalunya (2014a): "Resolución 479/X por la que se acuerda presentar a la Mesa del Congreso de los Diputados la Proposición de ley orgánica de delegación en la Generalidad de Cataluña la competencia para autorizar, convocar y celebrar un referéndum sobre el futuro político de Cataluña". http://www.agenciaincat.la/wp-content/uploads/2014/01/Resolucio479_10_es.pdf [Consultado el 07/10/2014].

Parlament de Catalunya (2014b): "Decret 129/2014, de 27 de setembre, de convocatòria de la consulta popular no referendària sobre el futur polític de Catalunya”, Diari Oficial de la Generalitat de Catalunya, n. 6715. http://portaldogc.gencat.cat/utilsEADOP/PDF/6715A/1373016.pdf [Consultado el 06/10/2014].

Parlament de Catalunya (2014c): "Ley 10/2014, de 26 de septiembre, de consultas populares no referendarias y otras formas de participación ciudadana”, Diari Oficial de la Generalitat de Catalunya, n. 6715. http://portaldogc.gencat.cat/ utilsEADOP/PDF/6715/1373015.pdf [Consultado el 06/10/2014].

Porto, M. (2002): "Framing the world of politics: How governmental sources shape the production and the reception of TF news in Brazil”, 23rd International Conference of the International Association for Media and Communication Research. http://www.unb.br/fac/mporto/index.html [Consultado el 07/11/2007].

Presidencia de la Generalitat (2014): "Decreto 129/2014, de 27 de septiembre, de convocatoria de la consulta popular no refrendaria sobre el futuro político de Cataluña”. Diari Oficial de la Generalitat de Catalunya, n. 6715. http://portaldogc.gencat.cat/utilsEADOP/PDF/6715A/1373017.pdf [Consultado el 07/10/2014].

Scheufele, D. (1999): "Framing as a theory of media effects", Journal of Communication, n. 49 (1), pp. 103-122.

Scheufele, D. A., \& Iyengar, S. (2012): “The state of framing research: A call for new directions”, The Oxford Handbook of Political Communication Theories. New York: Oxford UniversityPress. http://www.researchgate.net/profile/Dietram 
Scheufele/publication/224818492_The_state_of_framing_research_A_call_for_new_directions/links/0fcfd510e9ad4631fa0 00000.pdf [Consultado el 17/02/2015].

Tábara, J. D. \& Costellá, M. (2001): Las culturas del agua en la prensa española. Los marcos culturales en la comunicación sobre el Plan Hidrológico Nacional. Barcelona: Universidad Autónoma de Barcelona.

Tribunal Constitucional (2014): “Impugnación de disposiciones autonómicas (Título V LOTC) promovida por el Gobierno de la Nación”. http://www.tribunalconstitucional.es/es/salaPrensa/Documents/NP_2014_074/P\%205830-2014.pdf [Consultado el 01/10/2014].

Vicente, M. y López, P. (2009): “Resultados actuales de la investigación sobre framing: sólido avance internacional y arranque de la especialidad en España”, Zer, n. 14 (26), pp. 13-34. http:/ /www.ehu.es/ojs/index.php/Zer/article/view/2750/2364 [Consultado el 10/07/2012].

Weaver, D. H. (2007): “Thoughts on agenda setting, framing and priming”, Journal of Communication, n. 57 (1), pp. 142-147.

Wikipedia (2014): “Consulta sobre el futuro político de Cataluña 2014”. http://es.wikipedia.org/wiki/Consulta_sobre_el_futuro_político_de_Cataluña_de_2014\#cite_ref-67 [Consultado el 08/10/2014]. 